\title{
Tropical diseases of the myocardium: a review
}

This article was published in the following Dove Press journal:

International Journal of General Medicine

7 April 2017

Number of times this article has been viewed

\author{
Zoe C Groom' \\ Aristotle D Protopapas ${ }^{2}$ \\ Vasileios Zochios ${ }^{3,4}$ \\ 'Costello Medical Consulting Limited, \\ Cambridge, ${ }^{2}$ Department of Surgery, \\ Division of Surgery and Cancer, \\ Imperial College London, London, \\ ${ }^{3}$ Department of Intensive Care \\ Medicine, Queen Elizabeth Hospital \\ Birmingham, University Hospitals \\ Birmingham NHS Foundation Trust, \\ ${ }^{4}$ College of Medical and Dental \\ Sciences, University of Birmingham, \\ Birmingham, UK
}

\begin{abstract}
Cardiovascular diseases are widely distributed throughout the world. Human parasitic infections are ubiquitous. Tropical parasites are increasingly recognized as causes of cardiovascular diseases. In this review, we address the most frequently reported parasites that directly infect the myocardium, including Trypanosoma cruzi, the protozoal causative agent of American trypanosomiasis (Chagas disease), and Taenia solium, the cestode causative agent of taeniasis and cysticercosis. We also discuss tropical endomyocardial fibrosis, trichinellosis and schistosomiasis. Health systems, attitudes, the perceptions of both patients and physicians as well as socioeconomic factors should all be explored and recognized as crucial factors for improving the control of cardiovascular diseases in the tropics. Clinicians throughout the world must remain aware of imported parasites as potential causes of cardiac diseases.
\end{abstract}

Keywords: cardiomyopathy, Chagas disease, myocardium, parasitic infection, tropical infections

\section{Introduction}

Cardiovascular diseases are widely distributed throughout the world. In developing countries, cardiovascular deaths are predicted to increase by 2020, and the importance of these diseases in low- and middle-income countries (LMICs) is becoming increasingly recognized. ${ }^{1}$ Based on 2008 data, the World Health Organization (WHO) estimated that $75 \%$ of the world's burden of cardiovascular disease was found in LMICs. ${ }^{2}$ In Africa alone, cardiovascular diseases account for approximately $15 \%$ of hospital admissions and $10 \%-20 \%$ of fatalities. ${ }^{3}$ While obesity and tobacco and alcohol consumption undoubtedly represent the major underlying causes responsible for the rise in cardiovascular diseases in LMICs, it is unlikely that they provide the full explanation for the increasing disease burden. ${ }^{1}$

In the tropics, cardiovascular diseases can be broadly divided into two groups as follows: 1) cardiovascular diseases endemic to the tropics and 2) cosmopolitan cardiovascular diseases. Diseases falling into Group 1 typically include more neglected causes of cardiovascular diseases and are generally exclusive to the world's poorest populations. Examples include Chagas heart disease and endomyocardial fibrosis (EMF), both of which are diseases specific to the tropics. ${ }^{4}$ Diseases falling into Group 2 include chronic noncommunicable diseases (CNCDs) such as arterial hypertension, rheumatic fever, pericarditis and ischemic heart disease. ${ }^{3}$

Cardiomyopathies can be defined as diseases of the myocardium associated with cardiac dysfunction. ${ }^{4}$ In developing countries, there appears to be an increased incidence of the "usual" forms of cardiomyopathy that have a modified clinical course,
Correspondence: Vasileios Zochios Department of Intensive Care Medicine, Queen Elizabeth Hospital Birmingham, University Hospitals Birmingham NHS Foundation Trust, Mindelsohn Way, Birmingham BI5 2TH, UK Email vasileioszochios@doctors.org.uk 
possibly arising as a result of genetic or environmental differences in these areas, such as malnutrition, pollution and pathogenic infections. ${ }^{4}$

Human parasitic infections in the tropics constitute a substantial, but neglected, burden of disease. Protozoan and helminth parasites can establish infections leading to a wide variety of clinical manifestations..$^{5}$ In this review, we discuss the importance of neglected tropical diseases (NTDs) as causes of cardiovascular morbidity (Table 1).

\section{Parasites and the myocardium}

Trypanosoma cruzi, the protozoal causative agent of American trypanosomiasis (Chagas disease), and Taenia solium, the cestode causative agent of taeniasis and cysticercosis, are parasites that preferentially infect the myocardium. Chagas disease, occurring in, but not limited to, the South Americas, is arguably the most relevant parasite to the myocardium.

\section{T. cruzi and American trypanosomiasis (Chagas disease)}

South American trypanosomiasis, or Chagas disease, is a vector-borne parasitic infection that is caused by the protozoan species T. cruzi. It is transmitted by triatomine bugs of the genera Triatoma, Panstrongylus and Rhodnius (Order Hemiptera; Family Reduviidae) (Figure 1). There are also minor vectors from 12 other genera that have been transmitting the disease among animals for almost 10 million years. ${ }^{6}$

Twenty-five million people living in Latin America are at risk of infection. During the acute stage of the infection, most infected individuals do not exhibit clinical signs, and it is therefore difficult to estimate the number of sufferers. ${ }^{7}$ Current estimates vary in the region, with 7-8 million people living with the parasite worldwide. ${ }^{8}$ Of those infected, it is estimated that as many as $30 \%$ will develop cardiac involvement

Table I Estimated prevalence of cardiovascular diseases caused by NTDs

\begin{tabular}{|c|c|c|}
\hline NTD & $\begin{array}{l}\text { Estimated number of } \\
\text { people with the } \\
\text { infection }\end{array}$ & $\begin{array}{l}\text { Number of cases with } \\
\text { heart involvement }\end{array}$ \\
\hline Chagas disease $^{6}$ & 7-8 million & $\begin{array}{l}30 \% \text { develop cardiac } \\
\text { involvement }\end{array}$ \\
\hline Cysticercosis $^{5,34}$ & 50 million & $\begin{array}{l}20 \%-25 \% \text { in patients with } \\
\text { concomitant documented } \\
\text { Neurocysticercosis }\end{array}$ \\
\hline $\mathrm{EMF}^{\prime}$ & 12 million & All \\
\hline Trichinellosis ${ }^{5,35}$ & 10,000 new cases yearly & $\begin{array}{l}21 \%-75 \% \text { of infected } \\
\text { patients }\end{array}$ \\
\hline Schistosomiasis' & 200 million & $>270,000$ \\
\hline
\end{tabular}

Abbreviations: EMF, endomyocardial fibrosis; NTD, neglected tropical disease.
A

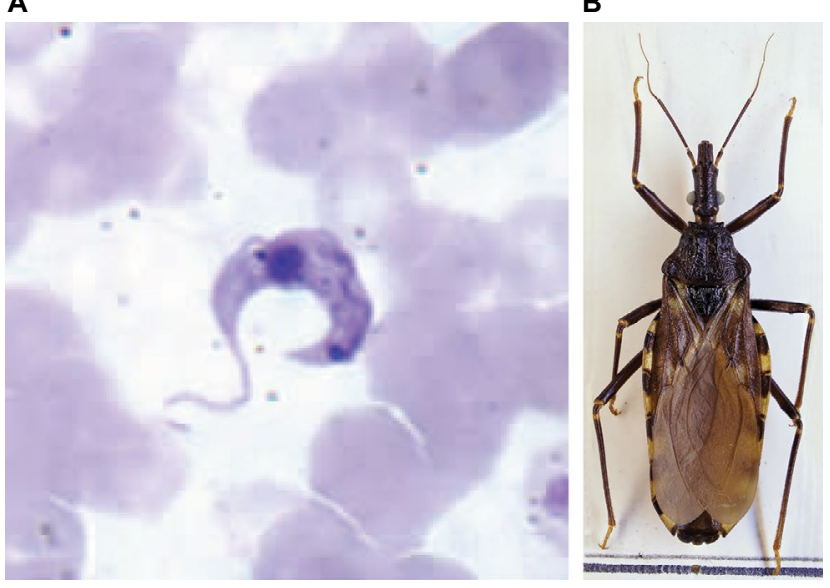

Figure I (A) Trypanosoma cruzi, the causative agent of Chagas disease. Reproduced from Centers for Disease Control and Prevention, part of the United States Department of Health and Human Services; 2004. Available from https://commons.wikimedia.org/ wiki/File:Trypanosoma_cruzi_B.jpg. ${ }^{36}$ (B) Triatoma infestans, the predominant vector of Chagas disease in the Southern Cone countries of South America. Reproduced from Wikipedians in Zoologische Staatssammlung München (ZSM); 20I4. Available from https://commons.wikimedia.org/wiki/File:Triatoma_infestans_-_ZSM.jpg...77

comprising heart failure, arrhythmias, mural thrombi (causing pulmonary and systemic emboli) or sudden death. ${ }^{4}$ Combined, these manifestations add to the complexity of the infection and increase the cost of patient management. ${ }^{9}$ The overall 5-year mortality for patients with established Chagas cardiomyopathy exceeds $50 \% .{ }^{4}$ As one of the world's most neglected diseases, drug development for American trypanosomiasis is severely lacking. Current therapies are associated with long treatment times, and risk of severe adverse reactions is high, being reported to occur in as many as $40 \%$ of treated patients. ${ }^{8}$

\section{Geographic distribution and life cycle of T. cruzi}

Chagas disease occurs in humans and a high number of wild and domestic animals. The parasite is widespread in Central and South America and the enzootic disease is endemic in $\sim 22$ countries, including all Latin American countries, with the exception of the Caribbean. ${ }^{6}$ The disease is restricted geographically to countries where its vector is endemic, ranging from South America to Mexico and southern USA. ${ }^{4}$

There are more than 130 species of triatomine bugs in the Americas, many of which are capable of being infected by, and transmitting, T. cruzi. ${ }^{10}$ Both adult and nymph stages of the insect life cycle are capable of transmitting the parasite. Female and male nymphs must take a blood meal in order to develop into adults, and female adults are required to take a blood meal in order to produce eggs. ${ }^{10}$ The life cycle of the parasite is shown in Figure 2.

Two epidemiological transmission cycles have been described based on the habitat preferences of the triatomine 


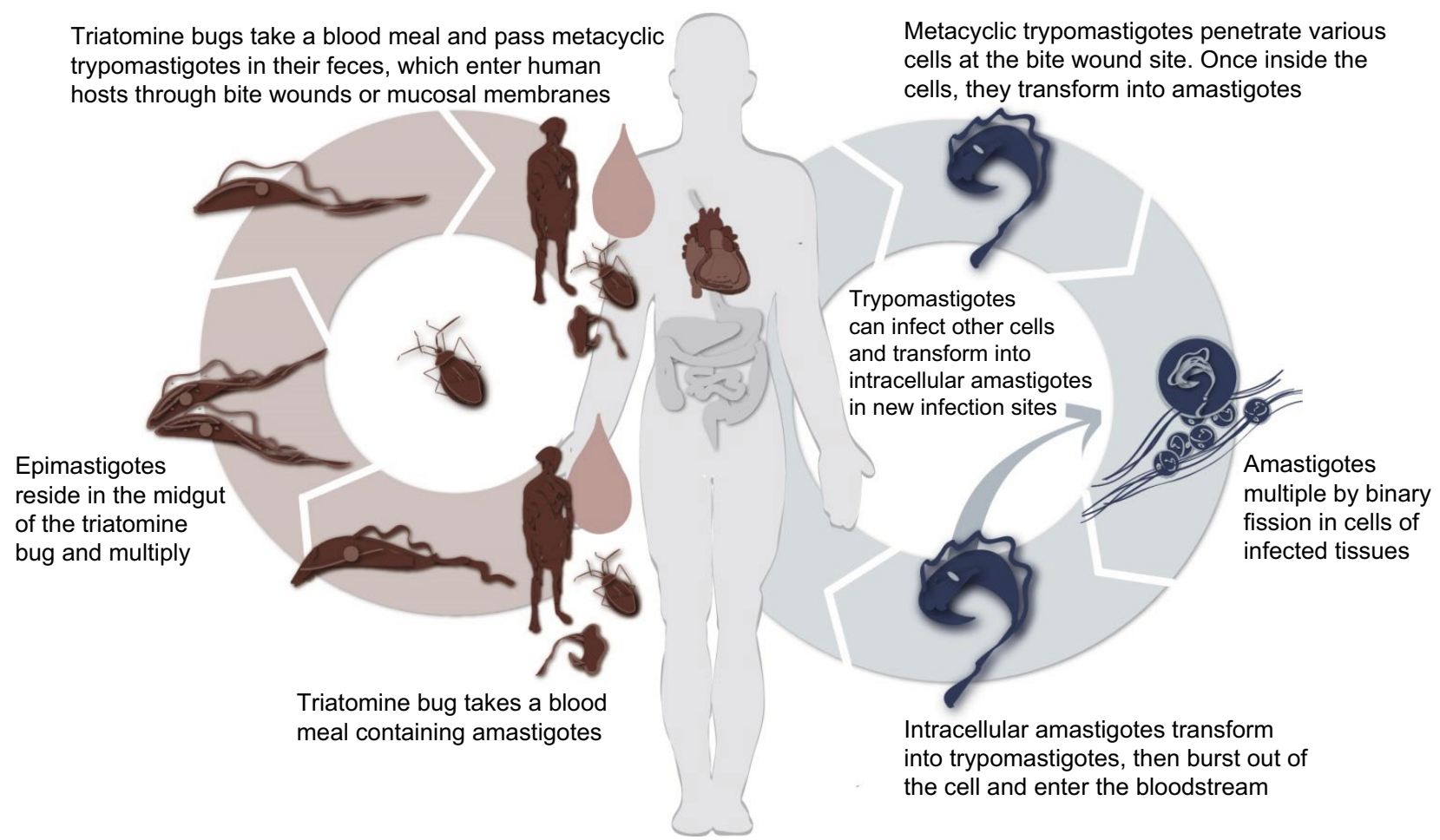

Figure 2 The life cycle of Trypanosoma cruzi.

Note: Adapted from Centers for Disease Control and Prevention. Available from: http://www.cdc.gov/media/DPK/20I4/docs/npi/Chagas_LifeCycle.pdf. ${ }^{38}$

vector: 1) sylvatic cycle and 2) domestic cycle (Figure 3). Transmission in sylvatic cycles involves wild animals that generally acquire the infection from vectors that preferentially colonize the nests of rodents or marsupial reservoir hosts. These vectors may then disperse into human dwellings, attracted by light, and there, they are capable of causing sporadic cases of human Chagas disease. ${ }^{10,11}$ The most common wild hosts of the parasite are rodents or marsupials; however, a large number of mammalian hosts may also harbor the parasite.

Many species of triatomine bugs may simply maintain infection among wild animals, but some species have adapted to living in human dwellings. In these situations, a domestic life cycle exists, and human infection occurs via transmission from domestic animals such as dogs and cats. The vectors in these domestic cycles can commonly be found in mud walls and thatched roofs of poor-quality housing in rural areas. As a result of this, Chagas disease is typically linked to socioeconomic status, and the rural poor are those that are most exposed. The domestic environment provides an abundant number of blood meal sources. ${ }^{10}$ In communities where the disease is endemic, $25 \%-100 \%$ of houses in a community may be infested, with single houses - and their immediate surroundings -supporting large colonies of juvenile and adult bugs. ${ }^{10} \mathrm{~A}$ single dwelling alone may harbor thousands of insects and as many as $50 \%$ of triatomine bugs in one household may be infected. An emerging concern, and a potential threat to vector control strategies, is the ability of some triatomine vector species to infest both domestic and sylvatic sites. These species have the potential to recolonize dwellings if vector control methods are relaxed. ${ }^{12}$

While vector transmission is the most common route of infection, Chagas disease may also be transmitted by blood transfusion, organ transplantation and congenital infection. ${ }^{5}$ Congenital infection can occur in up to $10 \%$ of women via ingestion of triatomine-contaminated food and drink. ${ }^{13}$

More recently, rural-to-urban and international migrations have changed the epidemiology of Chagas disease. Periurban and urban areas, areas of endemicity and areas of non-endemicity alike may all now have the potential to be associated with the parasitic infection. ${ }^{14}$ As a result of dynamic changes in the human population and the coordinated efforts of countries to interrupt vectorial and transfusional transmission, the prevalence and incidence of Chagas disease are constantly changing. ${ }^{5}$

\section{Disease stages}

There are two recognized stages of Chagas disease, the acute phase and the chronic phase. The chronic phase of the infection can be subcategorized into an indeterminate, 


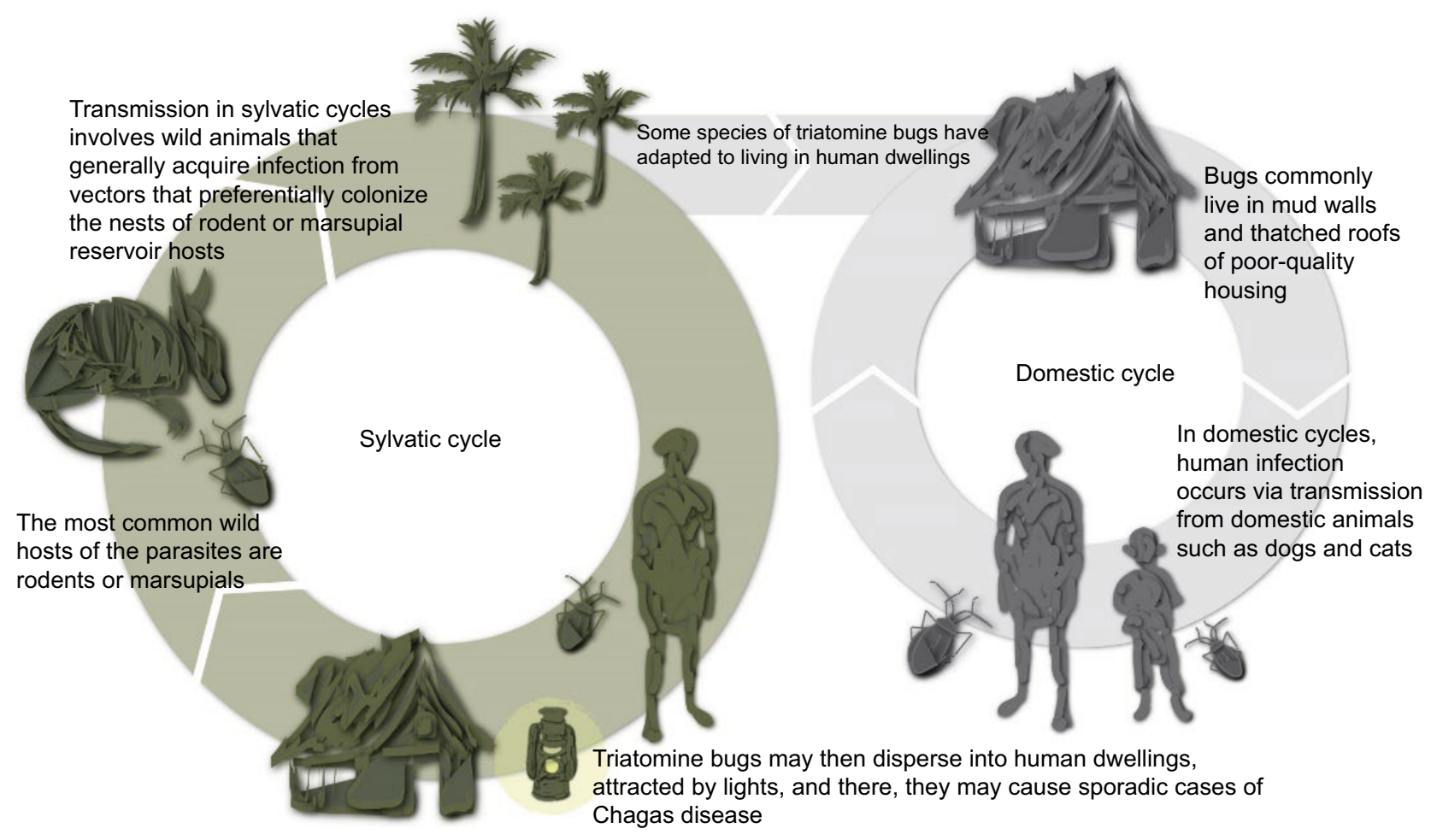

Figure 3 Sylvatic and domestic vector transmission cycles of the triatomine bug vector of Trypanosoma cruzi.

gastric or cardiac form. In the acute phase of the disease, cardiac involvement manifests as acute myocarditis; however, this is most often asymptomatic. During the indeterminate phase of infection, patients display evidence of $T$. cruzi infection without specific overt clinical evidence of cardiac or gastrointestinal disease. Approximately onethird to one-half of patients with indeterminate disease will eventually develop chronic Chagas cardiomyopathy (CCC). ${ }^{15}$ Figure 4 shows both the acute and chronic phases of Chagas disease.

During the acute phase of infection, which is most commonly observed in children but may occur at any age, infection is typically characterized by a self-limiting syndrome lasting between 4 and 8 weeks. ${ }^{4}$ Parasitemia is evident via direct smears. Approximately one-third of infected individuals show symptoms of infection, and when symptoms are present, they tend to be mild or resolve spontaneously. At the site of parasite entry, a chagoma may develop. If the site of entry was the conjunctiva, a characteristic unilateral periorbital edema can form called Romanãs sign. During this stage, there can be direct invasion of the myocardial fibers. Symptoms of the acute infection may include fever, malaise, lymphadenopathy, hepatosplenomegaly and acute myocarditis. ${ }^{4}$ In general, this stage of $T$. cruzi infection typically results in relatively mild illness. This mild illness, coupled with the fact that the rural poor are those at highest risk of infection and have limited access to health care, often means that individuals who are later identified as seropositive for the parasite do not tend to recall ever having had acute Chagas disease. ${ }^{16}$ It is estimated that the acute phase of infection has a $5 \%-10 \%$ mortality rate, with death most often a result of congestive heart failure due to severe myocarditis and/or meningoencephalitis, typically in children. ${ }^{4}$

Cellular and possibly humoral immune responses have been implemented in the eventual control of the acute stage of parasite infection. Nevertheless, these responses are not usually sufficient to result in the complete elimination of the parasite. ${ }^{5}$ As such, a variably long asymptomatic phase ensues, which is often referred to as the latent or indeterminate phase. The indeterminate phase of infection can be defined by a combination of the following: infection that is confirmed either serologically or parasitologically, normal chest radiograph and electrocardiogram (ECG), normal barium swallow and enema procedure and the absence of clinical signs and disease symptoms. ${ }^{17}$ While it is not fully understood why some patients in the indeterminate phase of T. cruzi infection go on to develop chronic Chagas disease, it is thought that several factors influence the course of disease. These include parasite strain, parasite load during the acute phase, the quality of the host immune response and the presence or absence of reinfection. 
A

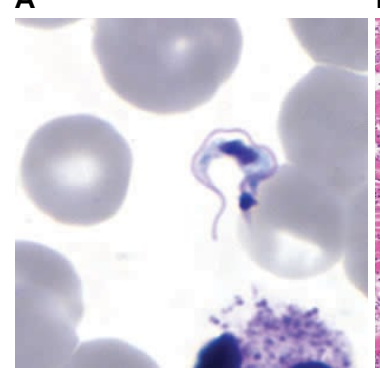

B

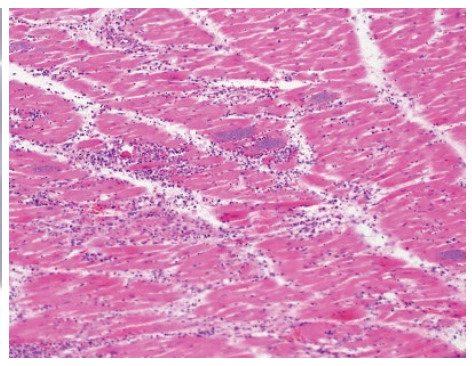

C

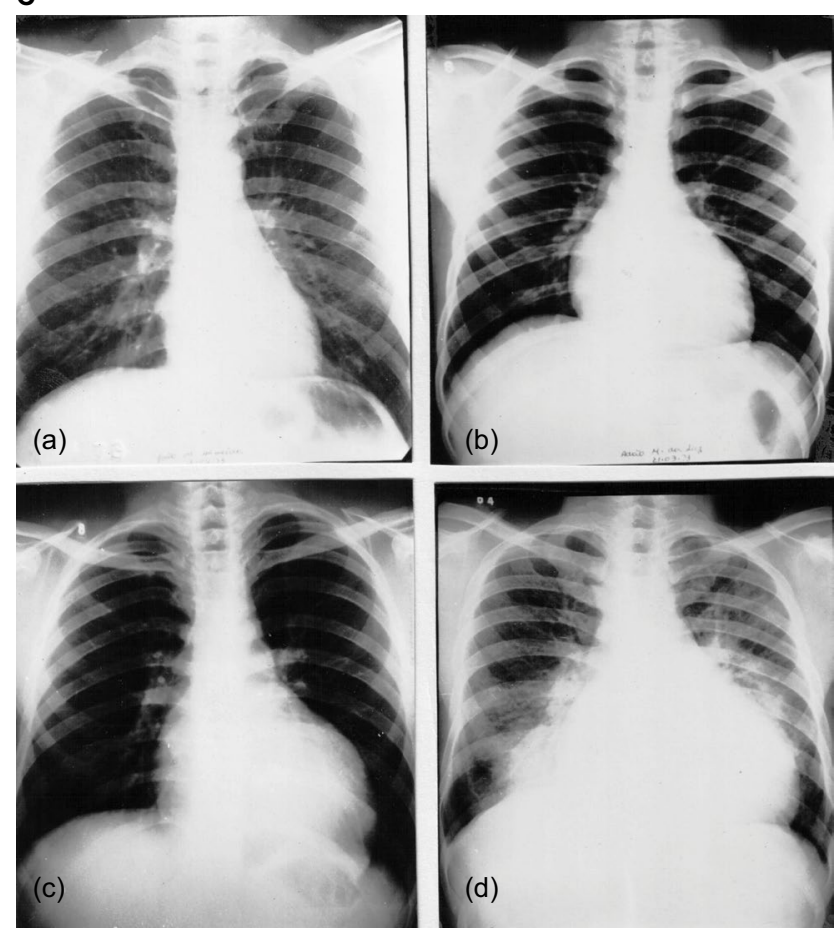

Figure 4 Acute and chronic phases of Chagas disease.

Note: Substituted with the following open access pictures. (A) Tryponosoma cruzi trypomastigote in a thin blood smear stained with Giemsa. Reproduced from DPDx - Laboratory Identification of Parasitic Diseases of Public Health Concern, Centers for Disease Control and Prevention, part of the United States Department of Health and Human Services. Available from https://www.cdc.gov/ dpdx/trypanosomiasisamerican/index.html. ${ }^{39}$ (B) Scar in the cardiac tissue of a 50-year-old male from South America who died suddenly and on whom an autopsy was performed. Hematoxylin/eosin-stained tissue sections of myocardium (I00x original magnification). Reproduced from Creepy Dreadful Wonderful Parasites, A parasitologist's view of the world, 20I I. Available from http://parasitewonders. blogspot.co.nz/201 I/02/case-of-week-150.html. ${ }^{40}$ (C) Cardiomegaly in chronic Chagas disease, as observed by chest $\mathrm{X}$-ray of 4 different patients with Chagas heart disease. (a) normal; (b) mild cardiomegaly; (c) moderate cardiomegaly; (d) severe cardiomegaly with pulmonary congestion. Copyright (C) 2007 Tarleton et al. Reproduced from Tarleton RL, Reithinger R, Urbina JA, et al. The Challenges of Chagas Disease— Grim Outlook or Glimmer of Hope? PLoS Med. 2007;4(I2):e332. ${ }^{41}$

Forms of chronic Chagas disease include cardiac, digestive and cardiodigestive infections. ${ }^{18}$ The most common and severe clinical manifestation is chronic cardiac Chagas disease (CCHD), progression to which can take several years or even decades. Chagas cardiomyopathy occurs in $20 \%-40 \%$ of chronically infected individuals, most commonly in those aged $20-59$ years. ${ }^{9}$ Due to the fact that the myopathy affects and incapacitates patients in their most productive working years, in addition to being associated with high morbidity and mortality, the disease is a major public health problem in Latin American countries. ${ }^{6}$

\section{Pathophysiology}

Typically, type 1 immune responses, driven by interleukin (IL)-12 and characterized by the production of interferon (IFN)- $\gamma$, are key for controlling T. cruzi infection. Due to the intracellular nature of the parasite, immune responses that reduce parasite replication within the host cells, such as the production of nitric oxide (NO) and lytic antibodies, have proven to be effective at controlling parasite numbers. ${ }^{19}$

CCC results from the combined effects of persistent parasitism, parasite-driven tissue inflammation, microvascular and neurogenic dysfunction, and autoimmune responses triggered by the infection. ${ }^{9}$ Individuals with this stage of infection tend to show a heightened T-lymphocyte type 1 response. Failure of immune control mechanisms, in particular, the expression of IL-10 and T-regulatory cells, is likely to result in unregulated inflammatory responses. Such responses, combined with persistent antigen stimulation, are likely to be what ultimately leads to extensive and irreversible tissue damage in CCHD. ${ }^{5}$

Geographically, and on an individual level, the spectrum of outcomes for patients infected with $T$. cruzi varies. ${ }^{5}$ This is likely a result of genetic differences in both the host and the parasite. For example, differences in the pathology of $T$. cruzi infection are thought to arise due to variations in the different parasite strains that are found in specific areas of South America. T. cruzi strains (Tcs) cluster into six discrete typing units (DTUs I-VI). ${ }^{20}$ Strains I and II are recognized as the two principal groups, both of which infect humans. TcII is associated with more severe disease and higher parasitemia. $\mathrm{TcI}$ is a major cause of Chagas disease in northern South America and Central America and prevails in sylvatic cycles throughout the continent. In contrast, in the Southern Cone region of South America, TcII, TcV and TcVI are implicated in Chagas disease. Currently, TcIII and TcIV have only been found to sporadically infect humans. ${ }^{21}$

The different strains of the parasite can be characterized molecularly using polymerase chain reaction (PCR)-based techniques. Studies involving PCR techniques have revealed a significant proportion of TcI parasites among Argentinian patients with $\mathrm{CCHD}^{22}$ and Columbian patients with chronic Chagas disease. ${ }^{23} \mathrm{TcII}, \mathrm{TcV}$ and TcVI have been identified in explant samples, and $\mathrm{TcV}$ and $\mathrm{TcVI}$ have been detected in samples obtained from patients with myocarditis reactivations. ${ }^{22}$ 


\section{Diagnosis}

\section{Clinical presentation}

The clinical presentation of the chronic cardiac stage of infection varies widely according to the extent of myocardial damage and manifests mainly as heart failure, cardiac arrhythmias and thromboembolism. ${ }^{9}$ Manifestations include palpitations, atypical chest pain, presyncope, syncope, dyspnea on exertion, congestive cardiac failure, symptomatic arrhythmias, thromboembolic disease, stroke and even sudden death. ${ }^{4}$ Specifically, the wall of the left cardiac ventricle becomes thinner, allowing the formation of an apical aneurysm, a distinctive feature of CCHD. Thrombi are often present in such cardiac aneurysms. Arrhythmias are also a hallmark of CCHD, resulting in heart block in the initial stages and malignant arrhythmias later. Sudden death in patients with CCHD may arise as a result of ventricular tachycardia, complete heart block or thromboembolic disease. The overall 5 -year mortality for patients with established CCC is $>50 \%{ }^{4}$

\section{Laboratory tests}

As with any parasitic infection, a lack of accurate diagnostic techniques means that infected individuals cannot be identified and therefore treated. The effectiveness of control campaigns, whether targeted at insect vectors, transmission blocking or vaccination of individuals, cannot be measured without competent diagnosticians and diagnostic techniques.

The type of technique implemented for the diagnosis of $T$. cruzi differs depending on the stage of infection that is under consideration. The acute stage of infection, where parasitemia is relatively high, may be best diagnosed through the use of microscopy and thick or thin blood film preparations. Concentration techniques can be used to aid in the diagnosis of infections that arise from low parasitemia. ${ }^{18}$

Due to low levels of parasitemia in the indeterminate and chronic phases, diagnosis has previously relied on serological tests, including enzyme-linked immunosorbent assay (ELISA) and indirect immunofluorescence assay (IFF). ${ }^{4}$ Patients are most typically diagnosed as seropositive through epidemiological studies or blood screening. Immunoglobulin $\mathrm{G}(\mathrm{IgG})$ and lifelong IgM responses are detectable through the use of serological tests; however, cross-reactivity with other parasitic infections and autoimmune diseases results in poor specificity. Furthermore, the majority of the currently available serological tests, whether developed in house or purchased commercially, use crude antigen preparations from inappropriate parasite life cycle stages, eg, the epimastigote stages, which are present in the insect vector but not in mammalian hosts. Serological tests often remain positive even after parasitological cure, thereby increasing the difficulty of making accurate estimations of disease burden. If appropriate facilities are available, xenodiagnosis or blood culture can be used to diagnose patients in the chronic stage of infection as an alternative to the more common serological techniques. Inoculation of blood samples into special media or into mice to demonstrate the presence of the parasite is not clinically useful because parasites may not be seen for several weeks. More recently, molecular-based assays using PCR techniques have been shown to be potentially sensitive for diagnosing patients with Chagas disease. They have also been shown to be the most effective way for evaluating cure following antitrypanosome treatment. ${ }^{4}$ The radioimmunoprecipitation assay based on iodinated $T$. cruzi proteins is sensitive and specific and is currently being used for confirmatory testing of many US blood donors and is approved by the US Food and Drug Administration. These assays, however, are not commercially available and whether they are more accurate than serological tests remains to be determined. ${ }^{9}$ The future development of new diagnostic techniques would benefit greatly from consensus on a diagnostic gold standard, without which, assessment of any newly developed techniques, in terms of their sensitivity and specificity, is impeded.

\section{Cardiac investigations}

In the acute stage of infection, the principal ECG abnormalities are first-degree atrioventricular (AV) block and QRS and T-wave changes. ${ }^{5}$ Infected individuals in the indeterminate phase of infection most often present no ECG or X-ray abnormalities; however, stress testing and echocardiography may disclose latent myocardial abnormalities. ${ }^{16}$

Echocardiography is therefore an important diagnostic modality for the initial assessment and long-term follow-up of patients with CCHD. ${ }^{16}$ Its use can facilitate the characterization and determination of predictors of mortality and clinical events. ${ }^{24}$ Left ventricular (LV) systolic function is usually preserved in the acute phase, unless the myocarditis leads to ventricular dysfunction. ${ }^{16}$ Advanced CCHD mimics chronic ischemic or idiopathic dilated cardiomyopathy. Characteristic echocardiographic findings include apical aneurysm, segmental LV contractile abnormalities and reduced LV systolic function. ${ }^{25}$ Pericardial effusion in acute stage infection occurs in $42 \%$ of patients. Doppler studies are also important. Furthermore, cardiac-gated magnetic resonance imaging (MRI) has emerged as an excellent technology for evaluating chagasic cardiomyopathy. Impaired LV function in echocardiography is the most consistent independent predictor of mortality. ${ }^{26}$

In an Argentinian study that enrolled 849 patients with chronic Chagas disease, LV systolic dimension and ejection 
fraction were found to be predictors of mortality. LV diastolic and systolic dimensions and ejection fraction were also predictors of nonfatal clinical events. ${ }^{24}$

\section{Management and control of American trypanosomiasis}

The management and control of American trypanosomiasis can be broadly categorized into the following: 1) treatment of different manifestations, including antiparasitic therapies, and 2) control of parasitic infection indirectly through vector control measures.

Antiparasitic drug development is challenging due to the intracellular nature of the parasite. There have been no new drugs developed for the treatment of Chagas disease since the $1970 \mathrm{~s}^{4}$ The publication of the T. cruzi genome in 2005 gave hope for the discovery of novel drug targets. ${ }^{27}$

Current drug therapies for the acute stage of infection include benznidazole and nifurtimox, which, although relatively effective in the acute stages of the infection, rely on patients completing a 60-day treatment course. The side effects associated with the therapy can be severe and reduce the chance that a patient will complete the full treatment course.

A Cochrane review of mortality and clinical progression of CCC after at least 4 years of follow-up identified 13 studies comparing the outcomes of 4,229 patients who received trypanocidal treatment (TT) or placebo. ${ }^{28}$ Results from this review revealed that receiving TT was associated with a $50 \%-90 \%$ smaller chance of having circulating antibodies or parasitic material, as compared with controls. The results on progression toward Chagas disease or death indicated smaller benefits. About one in five treated individuals abandoned their treatment, and one in 40 treated individuals had a severe reaction (needing hospitalization, additional treatments or interruption of this treatment). ${ }^{28}$ Most specialists and international institutions now recommend specific antitrypanosomal treatment for patients with chronic Chagas disease; however, better quality studies on patient management are necessary to ensure positive patient outcomes.

Cardiac involvement requires medical, interventional and surgical treatment. The use of $\beta$-blockers is safe and well tolerated..$^{9}$ Angiotensin-converting enzyme inhibitors are additionally used. Amiodarone is used to treat arrhythmias and has trypanocidal effects. The insertion of a pacemaker is also often necessary. Implantable cardioverter defibrillator (ICD) is used for patients with sustained ventricular tachycardia and those patients who have been successfully resuscitated from a near-sudden-death episode in the setting of LV dysfunction or those recovered from sudden cardiac death. The options of heart transplantation for end-stage congestive heart failure (reactivation of Chagas disease may arise as a result of antirejection immunosuppressive therapy) and stem cell-based therapy in very advanced stages of chagasic cardiomyopathy should be noted as well.

Control of Chagas disease through vector control measures has successfully been achieved in some areas of South America through the use of seroprevalence surveys that aim to identify areas most at risk of infection. Such areas may then be actively targeted through the use of vector control techniques such as pyrethroid insecticidal spraying and improvement of housing conditions. The elimination of cracks in mud walls and the provision of metal sheet roofing have proven to be simple yet effective techniques for reducing habitats of Chagas disease vectors. The "Southern Cone Initiative", initiated in the early 1990s, effectively brought about reduced T. cruzi transmission in many of the South American countries in which it was initiated. Vector control techniques used in the program included residual spraying with synthetic pyrethroids, housing improvements and health education. ${ }^{29}$ The success of this program was relatively dependent on the fact that the vector species of the disease in the countries in which the program was rolled out were Triatoma infestans and Rhodnius prolixus. These vectors can be described as "domiciliated", meaning their life cycles take place completely within domestic habitats. ${ }^{30}$

More recently, the Andean and Central American countries have adopted similar control programs plus universal screening of blood products to control transfusion-related infections. ${ }^{29}$ In these regions, the numbers of infected individuals remain higher than those of the Southern Cone countries. Possible explanations for this reduced effectiveness may include the fact that the vectors of disease in these areas are not domiciliated and can therefore reinfect dwellings from peridomestic and sylvatic habitats. ${ }^{30}$

New rural housing developments, labor-intensive and expensive insecticide spraying regimens and the fact that $T$. cruzi has many animal reservoirs inhibit the success of $T$. cruzi control programs. ${ }^{31}$ Continued vigilance and surveillance are required to prevent the resurgence of the disease in areas where disease prevalence has fallen.

\section{T. solium, taeniasis and cysticercosis}

T. solium, or pork tapeworm, is a cestode parasite, transmitted by eating raw or insufficiently cooked pork containing infective cysticercosis larvae. The parasite occurs most commonly in Southern Africa, China, India, Central America, Chile, Brazil, Papua New Guinea and non-Islamic South East Asia where human feces comes into contact with pigs and where pork may be eaten raw or undercooked. The parasite's life cycle is shown in Figure 5. 


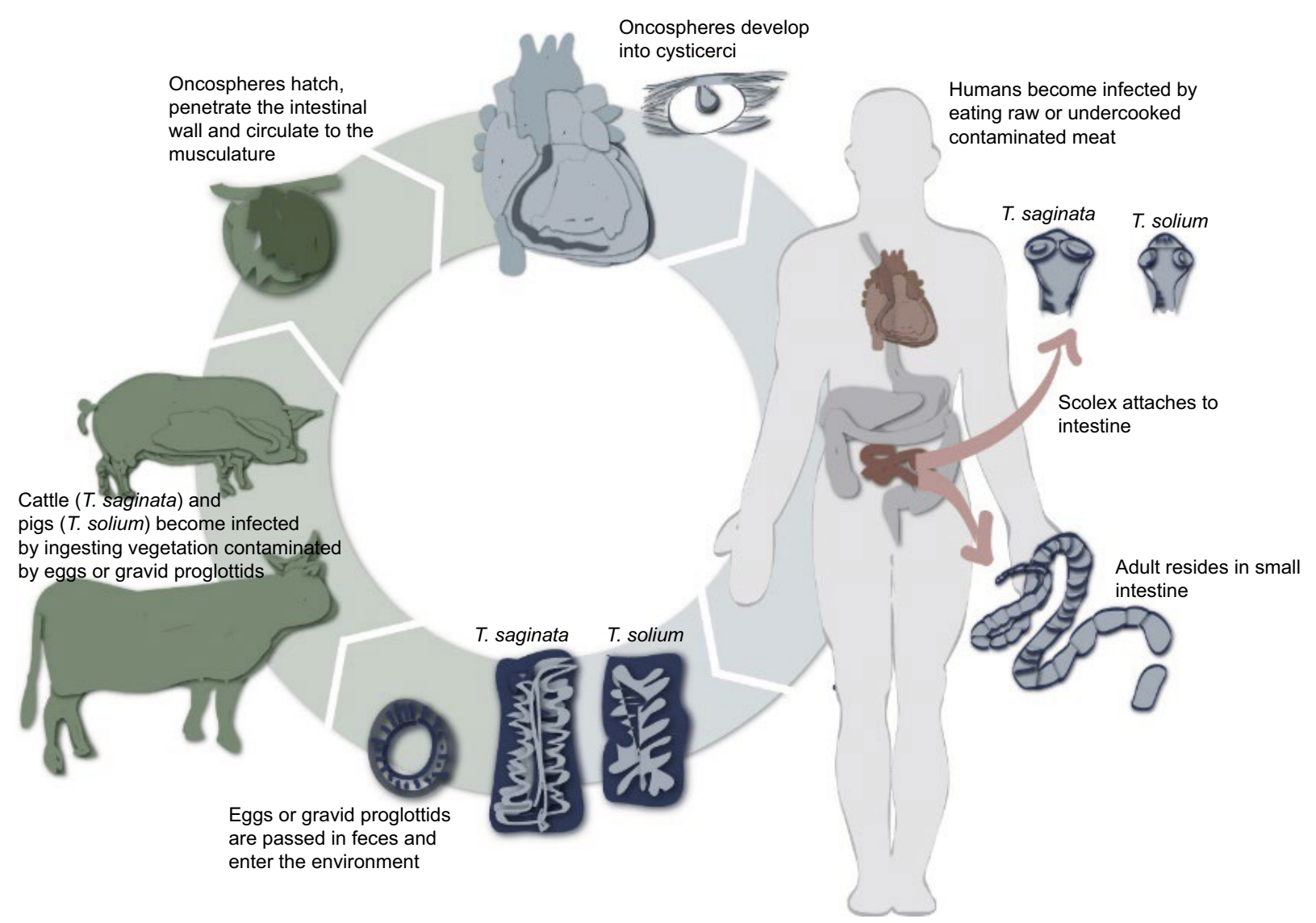

Figure 5 The life cycle of Taenia solium.

Note: Adapted from Centres for Disease Control and Prevention [webpage on the Internet]. Taenia Solium Life Cycle. 2016. Available from: http://www.cdc.gov/parasites/ taeniasis/biology.html. ${ }^{42}$

\section{Pathophysiology}

There are two diseases that may arise as a result of infection with either the tapeworm or the larval stages of the parasite, namely, taeniasis and cysticercosis, respectively. Taeniasis rarely produces serious side effects. Occasionally, there may be abdominal pain with intestinal disturbances and general loss of appetite. Cysticercosis is a much more serious disease and occurs as a result of infection via the ingestion of viable $T$. solium eggs that develop into larvae capable of causing cystic nodules in the subcutaneous tissues and muscles. Cerebral cysticercosis is considered the most serious complication; however, ocular, spinal, cutaneous, muscular or cardiac lesions may also present. The myocardial inflammatory response is variable and may result in granuloma formation and fibrosis. This may subsequently lead to arrhythmias and conduction abnormalities either spontaneously or during treatment. ${ }^{5}$ Cardiac involvement in cysticercosis has previously been thought to be rare, but autopsy studies have shown a prevalence of $20 \%-25 \%$ in patients with concomitant documented neurocysticercosis. ${ }^{5}$ Cardiac cysticercosis is often asymptomatic and is most commonly discovered during cardiac surgery or at autopsy.

\section{Diagnosis and treatment}

Diagnosis of the infection in general can be achieved through the use of fecal smears in which gravid segments or eggs may be visualized. The diagnosis of cysticercosis is typically carried out serologically, typically using ELISA. ${ }^{32}$ The cysts, when calcified, are visible by X-ray or computed tomography (CT) scan when in the brain.

Praziquantel (PZQ) is the medication most commonly used to treat active taeniasis. If the patient has cysticercosis in addition to taeniasis, PZQ should be used with caution due to the fact that it is cysticidal and can cause inflammation around dying cysts. If this occurs, it can lead to seizures. Niclosamide is an alternative treatment. The effective use of PZQ or surgery in cardiac cysticercosis is unclear and has not been directly investigated; however, these therapies are likely to be promising given their efficacy at other sites. ${ }^{5}$

\section{Other parasitic infections that may involve the myocardium}

Other parasites whose infection may not necessarily directly involve the myocardium but in which myocarditis is often present as an indirect consequence of the infection are as follows: 1) tropical EMF, a disease arising as a result of 
Table 2 Epidemiology, pathogenesis, clinical manifestations and treatment of parasitic infections that affect the myocardium

\begin{tabular}{|c|c|c|c|c|c|}
\hline Parasitic infection & Causative agent & $\begin{array}{l}\text { Geographic } \\
\text { distribution }\end{array}$ & $\begin{array}{l}\text { Mode of } \\
\text { transmission }\end{array}$ & Cardiac manifestation & Treatment \\
\hline \multicolumn{6}{|c|}{ Parasites that preferentially affect the myocardium } \\
\hline 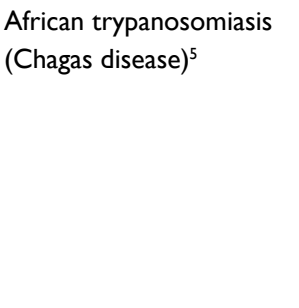 & Trypanosoma cruzi & $\begin{array}{l}\text { South and Central } \\
\text { America, Mexico and } \\
\text { Southern USA }\end{array}$ & $\begin{array}{l}\text { Vector-borne: } \\
\text { transfusional, } \\
\text { congenital, organ } \\
\text { transplant; food } \\
\text { borne: accidental }\end{array}$ & $\begin{array}{l}\text { Myocarditis, pericarditis } \\
\text { (acute phase); } \\
\text { cardiomyopathy (chronic } \\
\text { phase); heart failure, } \\
\text { heart block, ventricular } \\
\text { arrhythmias, apical } \\
\text { aneurysm, sudden cardiac } \\
\text { death }\end{array}$ & $\begin{array}{l}\text { Benznidazole or } \\
\text { nifurtimox }\end{array}$ \\
\hline Cysticercosis ${ }^{5}$ & Taenia solium & $\begin{array}{l}\text { Worldwide, but rural } \\
\text { areas in developing } \\
\text { countries have highest } \\
\text { rates of infection }\end{array}$ & Fecal-oral & Myocarditis: arrhythmias & $\begin{array}{l}\text { Albendazole or } \\
\text { praziquantel }\end{array}$ \\
\hline \multicolumn{6}{|c|}{ Miscellaneous infections of interest } \\
\hline $\begin{array}{l}\text { Tropical endomyocardial } \\
\text { fibrosis }^{43}\end{array}$ & No definitive cause & $\begin{array}{l}\text { Uganda, Ivory Coast, } \\
\text { Nigeria, Brazil and } \\
\text { India; the disease has } \\
\text { been occasionally } \\
\text { encountered outside } \\
\text { the tropics }\end{array}$ & $\begin{array}{l}\text { Etiology of EMF } \\
\text { remains unknown }\end{array}$ & $\begin{array}{l}\text { Pancarditis, ventricular } \\
\text { thrombi, atrioventricular } \\
\text { valve regurgitation and } \\
\text { heart failure }\end{array}$ & $\begin{array}{l}\text { No specific } \\
\text { treatment, invasive } \\
\text { procedures are } \\
\text { required in the acute } \\
\text { and chronic stages }\end{array}$ \\
\hline Trichinellosis ${ }^{5}$ & Trichinella spp. & Worldwide & Food borne & $\begin{array}{l}\text { Myocarditis, pericarditis } \\
\text { and arrhythmias }\end{array}$ & $\begin{array}{l}\text { Albendazole or } \\
\text { mebendazole }\end{array}$ \\
\hline Schistosomiasis ${ }^{5,18}$ & Schistosoma spp. & $\begin{array}{l}\text { Africa, South East Asia, } \\
\text { Central and South } \\
\text { America }\end{array}$ & Vector borne & $\begin{array}{l}\text { Right ventricular } \\
\text { hypertrophy, cor } \\
\text { pulmonale }\end{array}$ & Praziquantel \\
\hline
\end{tabular}

Abbreviation: EMF, endomyocardial fibrosis.

helminthic infection; 2) trichinellosis caused by the nematode Trichinella spiralis; and 3) schistosomiasis, a parasitic disease arising as a result of infection with the blood fluke Schistosoma spp. Schistosomiasis-associated pulmonary hypertension is well known. Furthermore, restrictive patterns of right ventricular dysfunction and EMF have been shown in autopsy studies in patients with advanced hepatosplenic schistosomiasis. ${ }^{5,18}$ These diseases are summarized in Table 2.

\section{Conclusion}

NTDs as contributors to cardiovascular diseases in LMICs in the tropics should not be ignored.

T. cruzi is arguably the most important neglected cause of cardiomyopathy in the tropics and one whose distribution in the future may expand. Accurate diagnosis and sustained interest in the research of these neglected diseases is of paramount importance. Until a thorough understanding of the contribution of NTDs to heart disease in LMICs is acquired, the success of appropriate intervention strategies, such as population screening and drug administration, cannot be guaranteed. Migration, population displacement and increased foreign travel have, in some cases, allowed the dispersal of parasitic diseases globally. As a result of the spread of certain neglected diseases, particularly those that are vector borne and subject to changes in distribution due to changes in the climate ${ }^{33}$ clinicians throughout the world must remain aware of "imported" parasites as potential causes of cardiac disease.

A potential result of the spreading distribution of NTDs may be a renewed and sustained interest in parasitic agents of cardiovascular disease. The success of control measures for the diseases discussed herein relies on continued investment in research into new treatments, prevention and novel control methods. Importantly, social determinants such as health systems, attitudes, the perceptions of both patients and physicians and socioeconomic factors should all be explored and recognized as crucial factors for improving the control of cardiovascular disease in the tropics.

\section{Acknowledgments}

The authors acknowledge the input of the Graphic Design team, Costello Medical Consulting Ltd, in particular Sarah Hope, who was the illustrator for many of the figures in this review, as well as Charlotte Bright and Courtney Toth for their editorial support in the design of the figures created for this paper.

\section{Disclosure}

The authors report no conflicts of interest in this work. 


\section{References}

1. Moolani Y, Bukhman G, Hotez PJ. Neglected tropical diseases as hidden causes of cardiovascular disease. PLoS Negl Trop Dis. 2012;6(6):e1499.

2. World Health Organization [webpage on the Internet]. Projections of Mortality and Burden of Disease, 2004-2030; Standard DALYs (3\% Discounting, Age Weights) - Baseline Scenario; 2008. WHO Regions; 2011. Available from: http://www.who.int/entity/healthinfo/global_burden_disease/DALY7_2008.xls. Accessed December 20, 2016.

3. Tomaszewski R. Cardiovascular diseases in the tropics. Prog Health Sci. 2011;1:217-219.

4. Yacoub S, Mocumbi AO, Yacoub MH. Neglected tropical cardiomyopathies: I. Chagas disease. Heart. 2007;94(2):244-248.

5. Hidron A, Vogenthaler N, Santos-Preciado JI, Rodriguez-Morales AJ, Franco-Paredes C, Rassi A. Cardiac involvement with parasitic infections. Clin Microbiol Rev. 2010;23(2):324-349.

6. Coura JR, Viñas PA [webpage on the Internet]. Chagas Disease: A New Worldwide Challenge. Outlook: Chagas Disease. 2010:S6-S7. Available from: www.nature.com/outlooks. Accessed March 08, 2017.

7. Tarleton RL, Reithinger R, Urbina JA, Kitron U, Gürtler GE. The challenges of Chagas disease - grim outlook or glimmer of hope. PLoS Med. 2007;4(12):e332.

8. Word Health Organization [webpage on the Internet]. Chagas Disease Fact Sheet. 2016. Available from: http://www.who.int/mediacentre/ factsheets/fs340/en/. Accessed December 20, 2016.

9. Ribeiro AL, Nunes MP, Telxeira MM, Rocha OC. Diagnosis and management of Chagas disease and cardiomyopathy. Nat Rev Cardiol. 2012;9(10):576-589.

10. Bern C, Kjos S, Yabsley MJ, Montgomery SP. Trypanosoma cruzi and Chagas disease in the United States. Clin Microbiol Rev. 2011;24(4):655.

11. Coura JR, Junqueira AC, Fernandes O, Valente SA, Miles MA. Emerging Chagas disease in Amazonian Brazil. Trends Parasitol. 2002;18(4): 171-176.

12. Gürtler RE. Sustainability of vector control strategies in the Gran Chaco region: current challenges and possible approaches. Mem Inst Oswaldo Cruz. 2009;104(suppl 1):52-59.

13. Massad E. The elimination of Chagas' disease from Brazil. Epidemiol Infect. 2008;136(9):1153-1164.

14. Moncayo A. Chagas disease: current epidemiological trends after the interruption of vectorial and transfusional transmission in the southern cone countries. Mem Inst Oswaldo Cruz. 2003;98(5):577-591.

15. Bestetti RB, Muccillo G. Clinical course of Chagas heart disease: a comparison with dilated cardiomyopathy. Int J Cardiol. 1997;60(2):187-193.

16. Tanowitz HB, Machado FS, Jelicks LA, et al. Perspectives on Trypanosoma cruzi-induced heart disease (Chagas disease). Prog Cardiovasc Dis. 2009;51(6):524-539.

17. Lescur FX, Le Loup G, Freilij H, et al. Chagas disease: changes in knowledge and management. Lancet Infect Dis. 2010;10(8):556-570.

18. Cheesbrough M. District Laboratory Practice in Tropical Countries. Second Edition Update. 2nd ed. Cambridge: Cambridge University Press; 2006.

19. Teixeira ARL, Hecht MM, Guimaro MC, Soussa O, Nitz N. Pathogenesis of Chagas disease: parasite persistence and autoimmunity. Clin Microbiol Rev. 2011;24(3):592-630.

20. Zingales B, Andrade SG, Briones MRS, et al. A new consensus for Trypanosoma cruzi intraspecific nomenclature: second revision meeting recommends TcI to TcVI. Mem Inst Oswaldo Cruz. 2009;104(7):1051-1054.

21. Who, how, what and where? Nature. 2010;465(n7301_supp):S8-S9. Available from: http://www.nature.com/nature/journal/v465/n7301_ supp/full/nature09222.html. Accessed March 08, 2017.

22. Burgos JM, Diez M, Vigliano C, et al. Molecular identification of Trypanosoma cruzi discrete typing units in end-stage chronic Chagas heart disease and reactivation after heart transplantation. Clin Infect Dis. 2010;51(5):485-495.
23. Ramirez JD, Guhl F, Rendon LM, Rosas F, Marin-Neto JA, Morillo CA. Chagas cardiomyopathy manifestations and Trypanosoma cruzi genotypes in chronic chagasic patients. PLoS Negl Trop Dis. 2010; 4(11):e899.

24. Viotti R, Vigliano C, Laucella S, et al. Value of echocardiography for diagnosis and prognosis of chronic Chagas cardiomyopathy without heart failure. Heart. 2004;90(6):655-660.

25. Acquatella H. Echocardiography in Chagas heart disease. Circulation. 2007;115(9):1124-1131.

26. Rassi A, Rassi A, Rassi SG. Predictors of mortality in chronic Chagas disease: a systematic review of observational studies. Circulation. 2007; 115(9):1101-1108.

27. El-Sayed NM, Myler PJ, Bartholomeu DC, Nilsson D, Aggarwal G, Tran AN. The genome sequence of Trypanosoma cruzi, etiological agent of Chagas disease. Science. 2005;309:409-415.

28. Villar JC, Perez JG, Cortes OI, et al. Trypanocidal drugs for chronic asymptomatic Trypanosoma cruzi. Cochrane Database Syst Rev. 2014; 5:CD003463.

29. Schofield CJ, Dias JC. The southern cone initiative against Chagas disease. Adv Parasitol. 1999;42:1-27.

30. Gourbière S, Dumontiel E, Rabinovich JE, Minkoue R, Menu F. Demographic and dispersal constraints for domestic infestation by nondomicilated Chagas disease vectors in the Yucatan Peninsula, Mexico. Am J Trop Med Hyg. 2008;78(1):133-139.

31. Ceaser M. Battling with Chagas disease in South America. Lancet Infect Dis. 2005;5:470-471.

32. Garcia HH, Parkhouse RM, Gilman RH, et al. Serum antigen detection in the diagnosis, treatment, and follow-up of neurocysticercosis patients. Trans R Soc Trop Med Hyg. 2000;94(6):673-676.

33. Nouvellet $P$, Dumonteil E, Gourbière S. The improbable transmission of Trypanosoma cruzi to human: the missing link in the dynamics and control of chagas disease. PLoS Negl Trop Dis. 2013;7(11):e2505.

34. Kraft R. Cysticercosis: an emerging parasitic disease. Am Fam Physician. 2007;76(1):91-96.

35. Pozio E, Foggin CM, Gelanew T, et al. Trichinella zimbabwensis in wild reptiles of Zimbabwe and Mozambique and in farmed reptiles of Ethiopia. Vet Parasitol. 2007;143(3-4):305-310.

36. Centers for Disease Control and Prevention, part of the United States Department of Health and Human Services; 2004. Available from https://commons.wikimedia.org/wiki/File:Trypanosoma_cruzi_B.jpg. Accessed December 20, 2016.

37. Wikipedians in Zoologische Staatssammlung München (ZSM); 2014. Available from https://commons.wikimedia.org/wiki/File:Triatoma_ infestans_-_ZSM.jpg. Accessed December 20, 2016.

38. Centres for Control of Disease. Chagas Disease: Life Cycle of Trypanosoma cruzi. 2016. Available from: http://www.cdc.gov/media/DPK/2014/ docs/npi/Chagas_LifeCycle.pdf. Accessed December 20, 2016.

39. DPDx - Laboratory Identification of Parasitic Diseases of Public Health Concern, Centers for Disease Control and Prevention, part of the United States Department of Health and Human Services. Available from https://www.cdc.gov/dpdx/trypanosomiasisamerican/index.html. Accessed December 20, 2016.

40. Creepy Dreadful Wonderful Parasites, A parasitologist's view of the world; 2011. Available from http://parasitewonders.blogspot. co.nz/2011/02/case-of-week-150.html. Accessed December 20, 2016.

41. Tarleton RL, Reithinger R, Urbina JA, et al. The Challenges of Chagas Disease - Grim Outlook or Glimmer of Hope? PLoS Med. 2007;4(12):e332.

42. Centres for Disease Control and Prevention [webpage on the Internet]. Taenia Solium Life Cycle. 2016. Available from: http://www.cdc.gov/ parasites/taeniasis/biology.html. Accessed December 20, 2016.

43. Mocumbi AOH, Falase AO. Recent advances in the epidemiology, diagnosis and treatment of endomyocardial fibrosis in Africa. Heart. 2013;99(20):1481-1487. 
The International Journal of General Medicine is an international, peer-reviewed open-access journal that focuses on general and internal medicine, pathogenesis, epidemiology, diagnosis, monitoring and treatment protocols. The journal is characterized by the rapid reporting of reviews, original research and clinical studies across all disease areas.
The manuscript management system is completely online and includes a very quick and fair peer-review system, which is all easy to use. Visit http://www.dovepress.com/testimonials.php to read real quotes from published authors. 